\title{
PÓS-OPERATÓRIO DE TRANSPLANTE RENAL: AVALIANDO O CUIDADO E O REGISTRO DO CUIDADO DE ENFERMAGEM
}

\author{
Post Operation of Renal Transplant: \\ Evaluating the Care and the Nursing Care Record \\ Post Operatorio de Trasplante Renal: \\ Evaluación del Cuidado y el Registro del Cuidado de Enfermería
}

Keroulay Estebanez Roque

Enirtes Caetano Prates Melo

Teresa Tonini

\begin{abstract}
Resumo
Objeto foi avaliação do cuidado e do registro de enfermagem no pós-operatório de transplante renal. Os objetivos foram identificar os cuidados de enfermagem registrados nos prontuários de clientes no pós-operatório de transplante renal; discutir as implicações dos registros para o cuidado de enfermagem e avaliar os cuidados de enfermagem. Trata-se de um estudo descritivo e observacional, realizado na Unidade de Transplante Renal de um hospital geral, localizado no Rio de Janeiro. Foi aprovado pelo Comitê de Ética e Pesquisa, e também houve a autorização da instituição pesquisada. Foram analisados 23 prontuários no período de maio a julho de 2005. Utilizou-se como instrumento de coleta de dados: formulário e observação sistematizada. Os resultados demonstram que o registro de enfermagem esteve presente nas 24 horas durante o período de internação, porém contemplava apenas a dimensão biológica do cuidado. As ações de cuidar ricas em subjetividade não foram registradas, apesar de ofertadas.
\end{abstract}

Palavras-chave: Controle de Formulários e Registros. Cuidados de Enfermagem. Avaliação.

\begin{abstract}
Object was evaluation of the care and the record of nursing in the post operative of renal transplant. The objectives were to identify the recorded cares of nursing in handbooks of customers in the post operative of renal transplant; to argue the implications of the records for the care of nursing and to evaluate the cares of nursing. It is about a descriptive and observational study, carried through in the Unit of Renal Transplant of a general hospital, located in Rio de Janeiro Brazil. It was approved by the Committee of Ethics and Research and also had the authorization by the searched institution. It was analyzed 23 handbooks in the period from May to July of 2005. It was used as instrument of data collection: form and systemize comment. The results demonstrated that the nursing record was present in the 24 hours during the period of internment, however contemplated only the biological dimension of the care. The actions to take care rich in subjectivity had not been recorded, although offered.
\end{abstract}

\section{Resumen}

El objeto del estudio fue la evaluación del cuidado y del registro del cuidado de enfermería en el post operatorio de trasplante renal. Los objetivos fueran identificar los cuidados de enfermería registrados en los prontuarios de clientes en el post operatorio de trasplante renal; discutir las implicaciones de los registros para el cuidado de enfermería y evaluar los cuidados de enfermería. Tratase de un estudio descriptivo y de observación, realizado en la Unidad de Trasplante Renal de un hospital general, situado en Rio de Janeiro - Brasil. Fue aprobado por el comité de Ética e Investigación y también hubo la autorización de la institución investigada. Fueron analizados 23 prontuarios en el período de Mayo hasta Julio de 2005. Fue usado como instrumento de la colecta de datos: formulario y observación sistematizada. Los resultados demuestran que el registro de enfermería estuvo presente en las 24 horas durante el período de internación, no obstante está contemplado solamente la dimensión biológica del cuidado. Las acciones de cuidar ricas en subjetividad, no fueran registradas, aunque ofrecidas.
Keywords:

Forms and Records Control. Nursing Care. Evaluation.
Palabras clave:

Control de Formulariosy Registros. Atención de Enfermería. Evaluación. 


\section{INTRODUÇÃO}

Este estudo derivou de uma pesquisa realizada entre 2005 e 2006, sobre a efetividade do cuidado de enfermagem. Essa avaliava a qualidade do cuidado de enfermagem ofertado aos clientes submetidos ao transplante renal.

0 cuidado de enfermagem não se restringe apenas à dimensão biológica, este também abarca a dimensão subjetiva. Levam-se em conta as subjetividades circulantes na interação entre os sujeitos envolvidos no ato de cuidar: enfermeira/cliente/família.

Cuidar é, portanto, mais que um ato. É uma atitude para além de um momento de atenção, zelo e desvelo. A atitude de cuidado representa ocupação, preocupação, responsabilização e envolvimento afetivo com o outro. 0 cuidado encontra-se na essência do ser humano como um modo-de-ser fundamental ao homem. Quando cuidamos, é necessário entrar em sintonia com quem está sendo cuidado, através da intimidade, do respeito, da escuta, da delicadeza e do ritmo'.

0 cuidado é uma ação incondicional do trabalho de enfermagem que envolve movimentos corporais ricos em impulsos de amor, ódio, alegria, tristeza, esperança, desespero, energia e disponibilidade para agir, tocar e sentir odores. Cuidado é um ato libertador que transcende às práticas e emoções, representando a essência da enfermagem².

Uma das responsabilidades da enfermeira é auxiliar o cliente em sua vivência diária ou com atividades que rotineiramente executaria sem assistência, como por exemplo: respirar, comer, eliminar, repousar, dormir, locomover-se, cuidar da higiene pessoal, vestir-se adequadamente. A enfermeira deve também propiciar atividades sociais, aprendizagem, ocupação recreativas e produtivas. Enquanto ela presta cuidados básicos de enfermagem, tem oportunidade de estar atenta ao que o cliente expressa, tomar interesse por ele/ família, avaliar suas necessidades e estabelecer relações interpessoais essenciais a uma enfermagem eficiente ${ }^{3}$.

A assistência de enfermagem tem se focado em ações que buscam atender as necessidades do cliente/família, não apenas utilizando dados clínicos e executando procedimentos técnicos.

Desta forma, a assistência de enfermagem pode ser sistematizada através da abordagem de identificação e resolução de problemas, exigindo que o enfermeiro desenvolva habilidades cognitivas, interpessoais e técnicas, no sentindo de atender as necessidades do ser humano que esta sendo cuidado. 0 processo de enfermagem consiste em cinco fases seqüenciais e inter-relacionadas: histórico, diagnóstico de enfermagem, planejamento, implementação e avaliação 4,5,6.

Devemos ressaltar que todas as fases do processo de enfermagem devem ser documentadas através do registro de dados nos prontuários. Este pode ser definido como documento único, que concentra todas as informações relativas à saúde de cada cliente, evidenciando as alterações e demonstrando a sua evolução durante todo o período do cuidado de enfermagem? .

0 prontuário do cliente é destacado como um dos importantes instrumentos na atenção à saúde na medida em que proporciona um registro documentado do processo de tomada de decisão. Os registros de informação e os planos relacionados com os problemas identificados pelos enfermeiros devem focalizar o cliente (o que não parece ocorrer) e ser dispostos de modo a compor um "sistema lógico" de desenvolvimento. 0 prontuário pode ser utilizado para várias finalidades: acompanhamento sistematizado do cliente, avaliação da assistência, auditoria, fonte de informação para coleta de dados para estudos e pesquisas.

A característica do registro varia de um profissional para o outro, e, por esse motivo, cada profissão propõe modelos diferenciados. Nesse aspecto, formas/formatos pouco importam na medida em que a qualidade da informação é que faz toda a diferença. Em linhas gerais, fala-se de clareza, concisão, legibilidade, veracidade como elementos fundamentais dessa ação. Mas parece que aí reside o nosso descompasso. Por que utilizamos impresso e registros que se distanciam muito das necessidades do serviço e, sobretudo, do cliente? Por que não preenchemos vários campos dos impressos propostos? As informações destacadas nesses impressos não são relevantes? A ausência de registro e a elegibilidade do mesmo representam uma linguagem, um olhar que merece ser decodificado?

Entendemos que os registros de enfermagem são instrumentos formais da assistência e proporcionam visibilidade, implementação e continuidade do cuidado de enfermagem. Além disso, o registro possibilita o acompanhamento diário das condições do cliente, sendo uma forma de comunicação entre as equipes de enfermagem e multidisciplinar da própria unidade e de outros setores da instituição 8,9 .

Partindo do princípio que o cuidado é a razão de ser da enfermagem, torna-se imprescindível que o enfermeiro perceba as implicações do registro do cuidado nos prontuários. Porém, o registro precisa contemplar não apenas o cuidar realizado com intuito de satisfazer as necessidades físicas do cliente. É fundamental que os elementos subjetivos que emergem da ação de cuidar também sejam registrados; Conseqüentemente, um registro que privilegie informações que contêm esses elementos subjetivos do cuidado, além da dimensão "técnica", colabora para a qualidade da assistência prestada.

Todavia, temos percebido que, de um modo geral, a Enfermagem não tem dado o devido valor ao registro dos elementos subjetivos do cuidado. Mesmo nas unidades especializadas, como a Unidade de Transplante Renal, no qual o registro, geralmente, está presente e seu conteúdo é mais rico em informações, essa característica tem se mantido. Quando descremos os elementos de dimensão subjetiva do cuidado, estamos nos referindo aos momentos de fala, de escuta e interpretações entre a "cuidadora e o cliente".

Desta forma, este estudo traz contribuiç̃es para a reflexão crítica dos enfermeiros em relação à qualidade do cuidado e do registro de enfermagem prestados aos clientes inseridos neste contexto.

Neste sentido, definimos as seguintes questões norteadoras: 0 cuidado de enfermagem prestado aos clientes no pós-operatório de transplante renal é adequado? 0 registro de enfermagem expressa o cuidado prestado aos clientes no pós-operatório de transplante renal?

Para dar conta destas questões, estabelecemos os seguintes objetivos: identificar os cuidados de enfermagem registrados 
nos prontuários de clientes no pós-operatório de transplante renal; discutir as implicações dos registros para o cuidado de enfermagem; avaliar os cuidados de enfermagem.

\section{METODOLOGIA}

Trata-se de um estudo descritivo e observacional. 0 cenário da pesquisa foi uma Unidade de Transplante Renal de um Hospital Geral de grande porte (Hospital Y), vinculado ao Sistema Único de Saúde (SUS), localizado no Município do Rio de Janeiro. Foram analisados 23 prontuários de clientes submetidos a transplante renal no referido hospital no período de maio a julho de 2005. Os critérios de inclusão dos prontuários foram: os clientes terem sido transplantados no referido hospital, estarem na faixa etária de 18 a 64 anos, não apresentarem comprometimento cognitivo grave e concordarem em assinar o consentimento livre e esclarecido. 0 tempo médio de seguimento dos clientes foi de 15 dias, com variação de 9 a 38 dias.

A coleta de dados foi realizada a partir da observação sistematizada e, também, de formulário. A busca nos prontuários foi utilizada de forma complementar à observação sistematizada da assistência de enfermagem prestada aos clientes submetidos ao transplante renal.

A busca dos registros de enfermagem foi feita diariamente, durante todos os dias da semana, contemplando o período diurno e noturno. No entanto, a observação sistematizada foi feita somente no período diurno. Desta forma, o acompanhamento dos pesquisadores iniciava-se a partir do pré-operatório imediato até a alta hospitalar. 0 preenchimento dos formulários também foi feito diariamente.

0 estudo foi submetido e aprovado pelo Comitê de Ética e Pesquisa, atendendo as exigências da resolução 196/96, e também houve a autorização para a coleta de dados por parte da Unidade de Transplante Renal da instituição pesquisada. Os pesquisadores explicaram a necessidade da assinatura do Termo de Consentimento Livre e Esclarecido para os clientes e enfermeiros que participaram do estudo, bem como foi esclarecido que 0 anonimato dessas pessoas seria preservado. Para isso, utilizou-se um sistema de codificação, no qual os nomes foram substituídos pelas respectivas iniciais.

A estrutura básica do formulário constou de quatro partes. A primeira destinou-se aos dados coletados nos prontuários, contendo informações referentes à identificação do cliente (sexo, idade, grau de escolaridade, procedência, patologiabase que causou a insuficiência renal crônica, tipo de doador, tipo de terapia dialítica, tempo de terapia, dias de internação e antecedentes pessoais). Ainda foram coletados dados relacionados ao preenchimento do cabeçalho nos impressos; presença da evolução e prescrição de enfermagem durante o período de observação; assinatura do enfermeiro de forma legível e com número do COREN nos registros de enfermagem. A segunda parte correspondeu ao preenchimento das possíveis intercorrências no pós-operatório que foram observadas pelos pesquisadores, incluindo ainda espaço para descrição da ação de enfermagem e outro para completar se essa ação tinha sido registrada. A terceira está relacionada com a listagem de cuidados biológicos de enfermagem considerados essenciais no pós-operatório de transplante renal, possibilitando aos pesquisadores saber se esses cuidados estavam sendo realizados pela equipe de enfermagem. A quarta parte destinouse ao preenchimento da satisfação do cuidado de enfermagem pelos clientes, no momento da alta hospitalar. Na tentativa de quantificar a satisfação dos clientes, foi apresentada a eles uma série de ícone ${ }^{10}$ acompanhada da seguinte pergunta: Qual dessas imagens expressa melhor a sua satisfação em relação ao cuidado de enfermagem prestado durante o seu pósoperatório de transplante renal?

0 estudo baseou-se em dois pilares da avaliação da qualidade: o processo, que está relacionado com as atividades realizadas pelos provedores da assistência, tanto diagnóstica, como terapêutica e de reabilitação (a satisfação do cliente também é levada em conta no processo); e o resultado, referindo-se ao efeito sobre as ações e procedimentos que tiveram no estado de saúde dos clientes ${ }^{11}$.

Durante a etapa piloto da pesquisa, sentiu-se a necessidade de buscar um instrumento que permitisse sistematizar os resultados obtidos a partir da observação participante e da busca aos prontuários. Desta forma, foram elaborados três instrumentos de avaliação do cuidado de enfermagem prestado aos clientes submetidos ao transplante renal. Esses instrumentos envolviam variáveis que fizeram parte dos dados contidos no formulário, e foi apresentado um escore para cada variável. Os instrumentos foram intitulados de: Avaliação do prontuário, Avaliação do cuidado de enfermagem prestado no pós-operatório de transplante renal e Avaliação das intercorrências no pós-operatório de transplante renal. Os dois primeiros instrumentos de avaliação apresentaram uma pontuação de zero (0), cinco (5) ou dez (10). No terceiro, foi estabelecida uma pontuação de zero (0) ou dez (10).

Os instrumentos de avaliação do cuidado de enfermagem foram construídos com base em dois pilares da avaliação (processo e resultado), sofrendo uma primeira etapa de validação pelo método de Delphi com conjunto de três especialistas. 0 método de Delphi é uma técnica que busca o consenso de opiniões de um grupo de especialista a respeito de eventos futuros ${ }^{12}$. Foram escolhidos três critérios: viabilidade, validade e relevância. A viabilidade referese à facilidade operacional, dados necessários e outras barreiras associadas à aplicação do indicador, enquanto a validade é o grau com que $\mathrm{o}$ indicador alcança seus objetivos, ou seja, reflete o evento ou aspecto que aqui se propõe a medir. A relevância está relacionada à aplicabilidade do indicador. Durante o processo de avaliação desses instrumentos, cada especialista atribuiu pontos de um (1) a nove (9). Um ponto corresponde a uma avaliação extremamente negativa, $\mathrm{e}$ nove pontos, a uma avaliação extremamente positiva.

As variáveis envolvidas no instrumento de avaliação do prontuário foram: a) preenchimento do cabeçalho com identificação do cliente nos impressos; b) presença da evolução de enfermagem; c) assinatura do enfermeiro de forma legível e com número do COREN no dos registros dos cuidados de enfermagem; c) presença da prescrição de enfermagem diariamente (Tabela $1 \mathrm{em}$ anexo).

0 segundo instrumento de avaliação contemplou cuidados biológicos de enfermagem considerados essenciais no pós- 
operatório: a) manter repouso no leito nas primeiras 24 horas; b) monitorização contínua; c) pesar em jejum; d) medir diurese de $1 / 1$ hora nas primeiras 24 horas; e) verificar glicemia capilar; f) verificar sinais vitais; g) anotar a aceitação da dieta; h) manter cateter vesical pérvio; i) retirar cateter vesical no $4^{\circ}$ dia do $\mathrm{PO}$; j) realizar balanço hídrico; k) trocar curativo da ferida operatória; I) anotar aspecto da ferida operatória; m) realizar reposição volêmica; n) realizar medicações; o) orientar quanto imunossupressão; p) anotar eliminações intestinais (Tabela 2 em anexo ).

0 terceiro instrumento de avaliação (intercorrência no pósoperatório) buscou responder a três perguntas: a) Toda intercorrência no pós-operatório gerou uma ação de enfermagem? b) A ação de enfermagem foi adequada à intercorrência? c) A intercorrência e a ação de enfermagem foram registradas? (Tabela 3 em anexo).

0 cuidado de enfermagem não biológico, isto é, o cuidado prestado pela enfermeira a partir da sua subjetividade, foi observado durante todo o período do estudo e descrito em parte destinada no formulário de coleta de dados.

\section{RESULTADOS E DISCUSSÃO}

A idade dos clientes transplantados variou de 20 a 60 anos; verificou-se uma pequena predominância entre 0 sexo feminino $(52,2 \%)$. Os transplantes se concentram na faixa etária de 41 a 50 anos, tanto no sexo feminino quanto no masculino. A população estudada apresenta baixa escolaridade, $52 \%$ não concluíram o ensino fundamental.

A causa mais freqüente de insuficiência renal crônica foi hipertensão arterial sistêmica. Pesquisas mostram que as principais causas da insuficiência renal crônica são hipertensão arterial (24\%), glomerulonefrite $(24 \%)$ e diabetes melito $(17 \%)^{13}$.

0 tratamento mais freqüente da insuficiência renal crônica, prévio ao transplante, foi hemodiálise. No Brasil em 1999, 47.063 pacientes encontravam-se em diálise; destes, 53\% concentravamse na região sudeste. $90 \%$ dos pacientes $(n=42.355)$ realizavam hemodiálise, e o restante $(n=4.708)$ diálise peritoneal ${ }^{14}$.

0 tempo médio de diálise prévio ao transplante foi considerado alto (variou de 9 a 180 meses). 0 diagnóstico e o encaminhamento tardio para diálise são comuns em nosso meio, atingindo mais de $50 \%$ dos pacientes que iniciam diálise em São Paulo. A sobrevida dos pacientes com diagnóstico tardio é $18 \%$ inferior à daqueles com diagnóstico precoce. 0 maior risco de óbitos destes pacientes está relacionado à idade mais elevada e ao nível de desnutrição associado ao diagnóstico e encaminhamentos tardios ${ }^{14}$.

Espera-se que quanto maior for o tempo de diálise prétransplante, maior será o número de co-morbidades neste cliente. Possivelmente, este cliente apresentará um quadro clínico mais grave quando comparado a de outro que realizou o transplante renal logo após o diagnóstico de insuficiência renal terminal.

Houve o predomínio dos doadores vivos (56,5\%), principalmente parentes (43,5\%). Os receptores de rim doado por parente estiveram submetidos a um tempo menor de diálise pré-transplante. Os transplantados de rim doado por parente tiveram menor tempo de internação e menor aparecimento de complicações no pós-operatório.
Na maior parte dos casos estudados houve diurese imediata após o implante do rim (83\%). Dos três clientes que não urinaram, nas primeiras vinte e quatro horas, dois receberam rim cadavérico e um teve doação de parente. Os clientes que urinaram imediatamente tiveram um prognóstico melhor e com menor tempo de internação.

O tempo de internação mínimo foi de sete dias, verificando-se uma variação de 9 a 38 dias. 0 único cliente que permaneceu internado por mais de 30 dias foi um receptor de rim cadáver, com 108 meses (9 anos) de tratamento pré-transplante com hemodiálise, e sua patologia base para insuficiência renal terminal foi hipertensão arterial.

Nos prontuários analisados, o registro de enfermagem esteve presente nas 24 horas durante o período de internação, porém contemplava apenas uma parte do cuidado prestado ao cliente transplantado: a dimensão biológica. Aspectos considerados relevantes pela equipe de enfermagem eram sempre registrados, como os achados no exame físico. Percebeuse que os cuidados registrados não contemplavam integralmente o cuidado prestado a esses clientes. A observação mostrou que o cuidado ultrapassava os aspectos referentes ao corpo biológico. Os cuidados referentes às necessidades de conversas informais, apoio emocional e familiar, conforto, orientações, dúvidas e inquietudes dos clientes eram realizados, porém não foi dado o devido valor para o registro desses cuidados de enfermagem. A esfera subjetiva do cuidado de enfermagem ofertado ao cliente, e não registrado, se configura como uma ação de difícil "identificação e interpretação por parte das enfermeiras"?

0 contato entre a equipe de enfermagem e o cliente não é somente e objetivamente técnico; atitudes de consideração, como escutar, olhar atentamente para o cliente, manifestações de carinho e interagir com ele respondendo a perguntas informais, são momentos importantes no cuidar. Desta forma, a ocorrência desses momentos pode ser traduzida em interesse pela pessoa do cliente/família, transcendendo assim, a normatização do cuidado ${ }^{15}$.

Em todos os 23 prontuários dos clientes sob o seguimento, desde o pós-operatório imediato até a alta, as evoluções foram assinadas e carimbadas pelos enfermeiros, totalizando um escore igual dez. Considerando que o setor estudado não realiza prescrição de enfermagem, a avaliação desta variável foi pontuada como zero. Em relação ao preenchimento do cabeçalho das folhas de evolução, a pontuação estabelecida variou de zero a cinco, nenhum prontuário foi classificado dentro dos 10 pontos.

Toda intercorrência no pós-operatório imediato e mediato ocorrida com os clientes transplantados gerou uma ação de enfermagem correspondente em todos os clientes sob o seguimento. Todas as ações de enfermagem realizadas após intercorrências foram consideradas adequadas, atendendo às necessidades fisiológicas dos clientes. Estas intercorrências foram registradas pela enfermagem no prontuário do cliente. Porém, as ações de cuidar ricas em subjetividade não foram registradas, apesar de ofertadas.

Os principais cuidados ao corpo físico que deveriam ser prestados aos clientes no pós-operatório imediato e mediato de transplante renal foram realizados pela equipe de enfermagem. Conforme o instrumento de avaliação do cuidado proposto, estes critérios alcançaram 10 pontos. 
Durante o preenchimento do instrumento de coleta de dados e por meio da observação participante, identificamos que as enfermeiras extrapolam a oferta de cuidados marcados apenas pela racionalidade da doença ou de um corpo repartido por órgãos (pele, coração, pulmão, etc).

Há, seguramente, ações de cuidar que são entrelaçadas por uma rede de relações políticas, éticas, estéticas e subjetivas, que facilitam o processo de cura ou de estabilização do desequilíbrio fisiobiológico mental do cliente.

Os momentos de escuta e comunicação da enfermeira/ cliente/família foram ações de cuidar agenciadas por estes atores sociais. Estas são ações positivas ao conforto e à qualidade do cuidado. A nossa preocupação foi a inexistência de registros na evolução dos cuidados que a enfermeira presta a partir de sua subjetividade.

A ausência dos registros de enfermagem sobre o cuidado com o corpo-emocional dos clientes nos mostrou que as enfermeiras ainda não dão o devido valor ao registro desses elementos subjetivos do cuidado, apesar de realizar a todo tempo. Os momentos de falas, escutas, cumplicidades, confiança e esperança, nos quais há a produção de uma responsabilização em torno do problema que vai ser enfrentado e de relações de vínculo e aceitação do cliente, são silenciados pela falta desses elementos no registro de enfermagem.

0 silêncio, tanto quanto a palavra, tem condições de produção de significados. Ele não é transparente, mas ambíguo. Parafraseando Orlandi $^{16}$, o registro pode ser silenciador quanto ao que se escreve, muitas vezes se escreve para não se afirmar certas coisas e para não se dizer outras palavras. A palavra silenciadora impede a discussão e a discordância, e, conseqüentemente, as reflexões do saber-fazer da enfermagem.

Durante os cuidados relacionados à homeostase do corpo do cliente, foi imperativa a preocupação das enfermeiras com o estado emocional, as relaç̃̃es familiares e a capacidade de comunicação de seus clientes. As enfermeiras conversaram com os clientes sobre suas angústias, temores, desejos, afetos e notícias de esposa/filhos/netos. Sabe-se o quanto estas ações de cuidar colaboram para minimizar o quadro depressivo ou de apatia muito característico dos clientes em quadro clínico de risco. Além disso, a possibilidade do risco de morte é uma assombração para muitos dos clientes pós-transplante renal imediato, exigindo que a enfermeira esteja atenta também às suas necessidades espirituais, permitindo inclusive a presença de líderes da religião que ele professa. Estas ações de cuidar são fundamentais de serem registradas no prontuário do cliente.

0 cuidado de enfermagem prestado no pós-operatório foi considerado muito bom (74\%) e bom (26\%) pelos clientes durante toda a sua internação até a sua alta hospitalar. Porém, sabemos que essa avaliação feita pelos clientes em relação a sua satisfação tem limitações. A primeira limitação está relacionada à escolha de um único e amplo parâmetro para avaliar a satisfação dos clientes, que foi o cuidado de enfermagem. Outra limitação é que, de modo geral, quando o objetivo do cliente é alcançado, no caso, o transplante renal foi realizado com sucesso, se tem uma visão positiva do cuidado prestado.

\section{CONCLUSÃO}

Na unidade de transplante renal estudada, os registros de enfermagem estavam presentes nas 24 horas observadas durante o seguimento. No entanto, este registro contemplava apenas uma parte do cuidado de enfermagem prestado ao cliente transplantado. Isto, porém, não significa que os cuidados prestados se limitavam exclusivamente aos aspectos biológicos. Os enfermeiros registravam cuidados referentes às necessidades biológicas do corpo do indivíduo. No entanto, os elementos subjetivos do cuidado, como conversas informais, orientações, apoio, conforto e recreação, não foram registrados.

0 registro do cuidado de enfermagem não deve somente contemplar os aspectos objetivos da ação de cuidar. Esta ação é produzida por um corpo biológico racional-emocional e cognitivo-sensorial ${ }^{17}$. Em movimento, este corpo assume os jogos de linguagem, os signos e códigos de sua cultura e de sua profissão, influenciando e sendo influenciado pelo modo de perceber e de se relacionar com os corpos de seus clientes através das dimensões estéticas, sensibilidades, utopias e necessidades. Neste sentido, compreendemos que em uma ação de cuidar, por mais que se expresse o elemento/fator técnico, está presente 0 aspecto subjetivo. É esse aspecto/dimensão do cuidado que deixa de ser entendido como tal e também não é registrado no prontuário do cliente.

De alguma forma, ao deixar de registrar todos os processos que fazem parte da relação de cuidar, o enfermeiro desqualifica e banaliza alguns aspectos do cuidado que são identificados como componentes do seu cotidiano.

Sendo assim, é fundamental que a enfermeira registre 0 cuidado realizado com objetivo de proporcionar conforto, tranqüilidade, satisfação do cliente, que se encontra distante de seu meio ambiente familiar e social, possibilitando uma assistência livre de danos. Quando não registra, o enfermeiro deixa de cumprir os preceitos éticos e legais da profissão.

0 estudo permitiu observar que a equipe de enfermagem é comprometida com o cuidado e busca, sempre que possível, atender as necessidades biopsicossociais dos clientes transplantados. Outro fato importante são o maior quantitativo e a maior qualificação desses profissionais, já que é um cuidado específico ao agravo do cliente. Porém, a qualidade do cuidado prestado ao cliente renal crônico não deve se limitar apenas ao nível terciário, com tecnologias de alta complexidade, como 0 transplante renal. 0 processo de saúde deve cumprir o seu papel em todos os níveis dos serviços de saúde. 


\section{Referências}

1 Boff L. Saber cuidar: ética do humano; compaixão pela terra. $11^{\mathrm{a}}$ ed. Petrópolis (RJ): Vozes; 1999.

2 Figueiredo NMA, Machado WCA, Porto IS, Ferreira MF. A Dama de Branco Transcendendo para a Vida/Morte Através do Toque. In: Meyer DE, Waldow VR, Lopes MJM. Marcas da Diversidade: saberes e fazeres da enfermagem contemporânea. Porto Alegre (RS): Artes Médicas; 1998. p. 137-169.

3 Henderson V. Princípios básicos sobre cuidados de enfermagem. Brasília (DF): Cortez; 1988.

4 Yer PW, Taptich BJ, Bernocchi-Losey D. 0 processo de enfermagem. Tradução de Regina Machado Garcez. In: . Processo e diagnóstico em enfermagem. Porto Alegre (RS): Artes Médicas; 1993. 5 Potter PA, Perry AG. Implementação do processo de enfermagem. In:___ Grande tratado de enfermagem prática: clínica e prática hospitalar. $3^{\mathrm{a}}$ ed. São Paulo (SP): Santos; 2002. p. 101-122.

6 Smeltzer SC, Bare BG. Reflexão crítica e o processo de enfermagem. In: . Brunner e Suddarth Tratado de enfermagem médico-cirúrgica. $8^{a}$ ed. Rio de Janeiro (RJ): Guanabara Koogan; 1998. p. 22-30.

7 França TMR, Chicanel MS. Por que cuidar do prontuário? In: Figueiredo NMA. Diagnóstico de enfermagem: adaptando a taxonomia a realidade. São Paulo (SP): Difusão; 2004. p. 155-161.

8 Mazza VA. Intrumentalização para registrar em Enfermagem. In: Westhalen NE, Caccaro TE. Metodologia para a assistência de enfermagem: teorização, modelos e subsídios para a prática. Goiânia (G0): AB; 2001. 9 Tanji S, Dahmer M, Oliveira SEM, Silva CMSLMD. A importância do registro no prontuário do paciente. Rev Enferm Atual 2003 nov; 4(24): 16-20.

10 Kloetzel K, Betoni AM, Campos VPG, Santos RN. Controle de qualidade em atenção primária à saúde. I: A satisfação do usuário. Cad. Saúde Pública 1998 jul; 14 (3): 623-628.
11 Donabedian A. A gestão da qualidade total na perspectiva dos serviços de saúde. Rio de Janeiro (RJ): Qualitymark; 1994.

12 Wright JTC, Giovinazzo RA. Delphi: uma ferramenta de apoio no planejamento prospectivo. Cad Pesquisa em Administração 2000 abr; 1(12): 54-65.

13 Sociedade Brasileira de Nefrologia (BR). Diretrizes em transplante renal. São Paulo (SP); 2001. Disponível em: http://www.sbn.org.br.

14 Sesso RCC. Epidemiologia da insuficiência renal crônica no Brasil. In: Ajzen H, Schor N. Guia de medicina ambulatorial e hospitalar de nefrologia. São Paulo (SP): Manole; p.1-7.

15 Castro ES, Mendes PW, Ferreira MA. A interação no cuidado: uma questão na enfermagem atual. Esc Anna Nery R Enferm 2005 abr; 9 (1): 39-45.

16 Orlandi EP. A fala de muitos gumos: as formas de silêncio. $4^{\mathrm{a}}$ ed. São Paulo (SP): Pontes; 1996.

17 Figueiredo NMA, Machado WCA. Ecosofia e Autopoiese no Cuidado com o Corpo. In: Santos I, organizadora. Enfermagem Fundamental: realidade, questões e soluções. São Paulo (SP): Atheneu; 2001. p. 191-210.

\section{Sobre as Autoras}

\section{Keroulay Estebanez Roque}

Enfermeira, Mestranda em Enfermagem pela Escola de Enfermagem Alfredo Pinto - UNIRIO-RJ. Professora do IBC/Unigranrio

\section{Enirtes Caetano Prates Melo}

Enfermeira, Doutora em Ciências pela Escola Nacional de Saúde Pública - FIOCRUZ. Professora Adjunta I do DESP/EEAP/UNIRIO-RJ.

\section{Teresa Tonini}

Enfermeira, Doutora em Saúde Coletiva pelo IMS/UERJ. Professora Adjunta I do DEF/EEAP/UNIRIO-RJ.

\section{ANEXOS}

Tabela 1: Modelo de Instrumento de Avaliação do Prontuário

\section{AVALIAÇÃO DO PRONTUÁRIO}

\begin{tabular}{l|l}
\hline & $\begin{array}{l}0=0 \text { prontuário está preenchido incompleto em mais da metade dos dias observados } \\
\text { A. Preenchimento do Prontuário }\end{array}$ \\
\hline \multirow{2}{*}{$\begin{array}{l}10=0 \text { prontuário está completo em mais da metade dos dias observados. } \\
\text { B. Evolução de Enfermagem }\end{array}$} & $\begin{array}{l}0=\text { Não há evolução de enfermagem no prontuário. } \\
5=\text { Há evolução de enfermagem (diurna e noturna) em maior parte dos dias observados. } \\
10=\text { Há evolução de enfermagem (diurna e noturna) em todos os dias observados. }\end{array}$ \\
\hline C. Assinatura do Enfermeiro & $\begin{array}{l}0=\text { Não há assinatura e carimbo nas evoluções. } \\
5=\text { Há assinatura e carimbo na maior parte das evoluções. } \\
10=\text { Há assinatura e carimbo em todas as evoluções. }\end{array}$ \\
\hline & $\begin{array}{l}0=\text { Não existe prescrição de enfermagem implementada no setor. } \\
5=\text { A prescrição de enfermagem não é realizada diariamente. } \\
10=\text { A prescrição de enfermagem é diária. }\end{array}$ \\
\hline
\end{tabular}


Tabela 2: Modelo de escala do pós-operatório imediato e mediato

\section{CUIDADOS DE ENFERMAGEM NO PO IMEDIATO E MEDIATO}

\begin{tabular}{|c|c|}
\hline A. Repouso no leito & $\begin{array}{l}0=\text { Cliente não foi mantido em repouso por } 24 \mathrm{~h} . \\
5=\text { Cliente foi mantido em repouso por menos de } 24 \mathrm{~h} . \\
10=\text { Cliente foi mantido em repouso de } 24 \mathrm{~h} .\end{array}$ \\
\hline B. Monitorização Contínua & $\begin{array}{l}0=\text { Não há nenhum aparelho de monitorização cardíaca no setor. } \\
5=\text { A monitorização cardíaca é por menos de } 24 \mathrm{~h} . \\
10=\text { A monitorização cardíaca é nas primeiras } 24 \mathrm{~h} .\end{array}$ \\
\hline C. Pesar em jejum & $\begin{array}{l}0=\text { Cliente não é pesado em jejum diariamente. } \\
5=\text { Cliente é pesado em jejum porém não é diariamente. } \\
10=\text { Cliente é pesado em jejum diariamente. }\end{array}$ \\
\hline D. Medir diurese & $\begin{array}{l}0=\text { Diurese não é medidas. } \\
5=\text { Diurese é medida } 4 \text { vezes ao dia,mesmo nas primeiras } 24 \mathrm{~h} . \\
10=\text { Diurese é medida de } 1 \text { em } 1 \text { hora nas primeiras } 24 \mathrm{~h} \\
\text { e posteriormente } 4 \text { vezes ao dia. }\end{array}$ \\
\hline E. Verificar glicemia capilar & $\begin{array}{l}0=\text { A glicemia capilar não é verificada quando prescrita. } \\
5=\text { A glicemia de capilar não é verificada de } 6 \text { em } 6 \text { horas conforme prescrito. } \\
10=\text { A glicemia de capilar é verificada de } 6 \text { em } 6 \text { horas conforme prescrito. }\end{array}$ \\
\hline F. Verificar Sinais Vitais & $\begin{array}{l}0=\text { Os sinais vitais não são aferidos. } \\
5=\text { Os sinais vitais não são aferidos de } 1 \text { em } 1 \text { hora nas primeiras } 24 \mathrm{~h} . \\
10=\text { Os sinais são aferidos de } 1 \text { em } 1 \text { hora na primeiras } 24 \mathrm{~h} \text {, } \\
\text { posteriormente de } 6 \text { em } 6 \text { horas. }\end{array}$ \\
\hline G. Anotar a aceitação da dieta & $\begin{array}{l}0=A \text { aceitação da dieta não é anotada. } \\
5=\text { A aceitação da dieta não é anotada diariamente. } \\
10=\text { A aceitação da dieta é anotada diariamente. }\end{array}$ \\
\hline H. Manter cateter vesical pérvio e fixado & $\begin{array}{l}0=0 \text { cateter vesical não é mantido pérvio e fixado. } \\
5=0 \text { cateter vesical não é mantido pérvio e não fixado. } \\
10=0 \text { cateter vesical é mantido pérvio e fixado. }\end{array}$ \\
\hline J. Retirar cateter vesical no $4^{\circ}$ dia do PO & $\begin{array}{l}0=0 \text { cateter vesical não é retirado no } 4^{\circ} \text { dia de P0, } \\
\text { mesmo sem ocorrer nenhuma intercorrência. } \\
10=0 \text { cateter vesical é retirado no } 4^{\circ} \text { dia de PO. }\end{array}$ \\
\hline K. Realizar balanço hídrico & $\begin{array}{l}0=0 \text { balanço hídrico não é realizado. } \\
5=0 \text { balanço hídrico é realizado apenas nas primeiras } 72 \mathrm{~h} . \\
10=0 \text { balanço hídrico é realizado diariamente. }\end{array}$ \\
\hline L. Trocar curativo da ferida operatória & $\begin{array}{l}0=0 \text { curativo não é trocado diariamente. } \\
10=0 \text { curativo é trocado diariamente. }\end{array}$ \\
\hline M. Anotar aspecto da ferida operatória & $\begin{array}{l}0=0 \text { aspecto da feriada operatória não é anotado. } \\
5=0 \text { aspecto da feriada operatória não é anotado diariamente. } \\
10=0 \text { aspecto da feriada operatória é anotado diariamente } \\
\text { até retirar o curativo. }\end{array}$ \\
\hline N. Realizar reposição volêmica & $\begin{array}{l}0=\text { A reposição volêmica não é realizada segundo prescrição. } \\
5=\text { A reposição volêmica não é horária. } \\
10=\text { A reposição volêmica é horária. }\end{array}$ \\
\hline 0. Administrar medicações & $\begin{array}{l}0=\text { As medicações não foram administradas. } \\
5=\text { As medicações foram administradas porém em horários } \\
\text { diferentes dos aprazados. } \\
10=\text { As medicações foram administradas nos horários nos aprazados. }\end{array}$ \\
\hline P. Orientar quanto a imunossupressão & $\begin{array}{l}0=\text { Não foi realizada nenhuma orientação quanto imunossupressão. } \\
5=\text { A orientação quanto imunossupressão é realizada no momento da alta } \\
10=\text { A orientação quanto imunossupressão é realizada no decorrer } \\
\text { da internação até o momento da alta. }\end{array}$ \\
\hline Q. Anotar sobre eliminações intestinais & $\begin{array}{l}0=\text { As eliminações intestinais nunca são anotadas. } \\
5=\text { As eliminações intestinais não são anotadas. diariamente } \\
10=\text { As eliminações intestinais são anotadas diariamente. }\end{array}$ \\
\hline
\end{tabular}


Tabela 3: Modelo de Instrumento de Avaliação das Intercorrências no Pós-Operatório

\section{AVALIAÇÃO DAS INTERCORRÊNCIAS NO PÓS-OPERATÓRIO}

\begin{tabular}{l|l}
\hline A. Toda intercorrência no PO imediato e mediato gerou ação de enfermagem? & $\begin{array}{l}0=\text { Não, nenhuma } \\
10=\text { Sim, sempre. }\end{array}$ \\
\hline B. A ação de enfermagem foi adequada à intercorrência? & $\begin{array}{l}0=\mathrm{Não} . \\
10=\mathrm{Sim} .\end{array}$ \\
\hline C. A intercorrência e a ação de enfermagem foram registradas? & $\begin{array}{l}0=\mathrm{Não} . \\
10=\text { Sim. }\end{array}$ \\
\hline
\end{tabular}

